\title{
ASYMPTOTIC BEHAVIOUR OF SOLUTIONS TO NEUTRAL FUNCTIONAL DIFFERENTIAL EQUATIONS
}

\author{
Shaozhu Chen and Qingguang Huang
}

Sufficient or necessary conditions are established so that the neutral functional differential equation $\left[x(t)-G\left(t, x_{t}\right)\right]^{\prime \prime}+F\left(t, x_{t}\right)=0$ has a solution which is asymptotic to a given solution of the related difference equation $x(t)=G\left(t, x_{t}\right)+a+b t$, where $a$ and $b$ are constants.

\section{INTRODUCTION}

We shall mainly be concerned with the second order neutral functional differential equation (NFDE)

$$
\left[x(t)-G\left(t, x_{t}\right)\right]^{\prime \prime}+F\left(t, x_{t}\right)=0, \quad t \geqslant 0, \quad '=\frac{d}{d t},
$$

where $G$ and $F$ are continuous functionals defined as follows.

For simplicity of notation, by $C J$ we denote the space $C(J, \mathbf{R})$ and by $\|\cdot\|$ the sup-norm of the space, where $J$ is any interval in $R$. Let $r(t) \geqslant 0$ be a continuous function on $[0, \infty)$ such that $t-r(t) \rightarrow \infty$ as $t \rightarrow \infty$; then $R(t)=\inf _{s \geqslant t}\{s-r(s)\}$ is finite for each $t \geqslant 0$ and nondecreasing. For any $\left.x \in C \mid R\left(t_{0}\right), L\right), 0 \leqslant t_{0}<L \leqslant \infty$, define $x_{t} \in C_{t}=C[-r(t), 0], t_{0} \leqslant t \leqslant L$, by $x_{t}(s)=x(t+s), s \in[-r(t), 0]$. For any $A, B \in\left[t_{0}, L\right), A<B$, set $\tau=\max _{A \leqslant t \leqslant B} r(t)$. We can always view $x_{t}$ for each $t \in[A, B]$ as the restriction of the function $x_{t}^{\tau}(s)=x(t+s), s \in[-\tau, 0]$ to the interval $[-r(t), 0]$, so the operator $x_{\bullet}:[A, B] \rightarrow C[-\tau, 0]$ is continuous. We then suppose that $G$ and $F$ are continuous in $t$, for fixed $t, G(t, \cdot)$ and $F(t, \cdot)$ are continuous functionals on $C_{t}$ and for any $A, B \in[0, \infty), A<B, G, F:[A, B] \times C[-\tau, 0] \rightarrow \mathbf{R}$ are continuous $\left(\tau=\max _{A \leqslant t \leqslant B} r(t)\right)$ and for any $t \in[A, B], u, v \in C[-\tau, 0]$,

$$
G(t, u)=G(t, v), \quad F(t, u)=F(t, v)
$$

provided $u(s)=v(s)$ for $-r(t) \leqslant s \leqslant 0$.

Received 7 November, 1988

Copyright Clearance Centre, Inc. Serial-fee code: 0004-9729/89 \$A2.00+0.00. 
A prototype of (1.1) is, say, the following NFDE with an unbounded delay $r(t)=$ $\max \left\{1, t^{1 / 2}\right\}$

$$
\left[x(t)-\frac{1}{2} x\left(t-t^{1 / 2}\right)\right]^{\prime \prime}+f(t) x(t-1)=0, \quad t \geqslant 0 .
$$

A function $x(t)$ is called a solution of $(1.1)$ if $x(t)$ is continuous on [ $\left.R\left(t_{0}\right), L\right)$ for some $t_{0} \geqslant 0, t_{0}<L \leqslant \infty$ and satisfies (1.1) on $\left[t_{0}, L\right)$. If $L=\infty$ in the above definition, then the solution $x(t)$ is called proper. For the fundamental theory of NFDE's the interested reader is referred to [2, Chapter 12].

In the particular case that $G \equiv 0$ the equation (1.1) degenerates into a delay equation

$$
x^{\prime \prime}(t)+F\left(t, x_{t}\right)=0 .
$$

If we assume further that $r(t) \equiv 0$, then (1.2) is reduced to an ordinary differential equation

$$
x^{\prime \prime}(t)+f(t, x)=0
$$

where $f(t, x)$ is continuous. Many authors have extensively studied the asymptotic behaviour of solutions to (1.3) (see [1] and the references cited therein). In [1], Chen obtains conditions for (1.3) to have solutions which are asymptotic to linear functions $a+b t$ with $b \neq 0$. The purpose of this paper is to generalise the results in [1] to NFDE (1.1). Our results will of course be valid for the delay differential equation (1.2).

In general, the asymptotic behaviour of solutions to NFDE (1.1) rests firmly on that of solutions to the difference equation

$$
D\left(t, x_{t}\right)=h(t),
$$

where $h(t)$ is continuous and $D\left(t, x_{t}\right)=x(t)-G\left(t, x_{t}\right)$ is the difference operator on the left-hand side of (1.1). We also note that in case $F \equiv 0$ each solution of (1.1) satisfies (1.4) with $h(t)=a+b t$; that is, the equation

$$
x(t)=G\left(t, x_{t}\right)+a+b t,
$$

where $a$ and $b$ are constants. The initial value problem for (1.4) at $t=t_{0} \geqslant 0$ is to find a continuous function $x(t)$ on $\left[R\left(t_{0}\right), L\right)$ for some $L, t_{0}<L \leqslant \infty$, such that $x\left(t_{0}+s\right)$ coincides with a given continuous function $u(s)$ on $\left[R\left(t_{0}\right), t_{0}\right]$ with $D\left(t_{0}, u_{t_{0}}\right)=h\left(t_{0}\right)$ and (1.4) is satisfied on $\left[t_{0}, L\right)$. If $L=\infty$, the solution defined above is also called a proper solution.

In Section 2 we shall give an existence result for proper solutions of (1.4). Some properties of solutions of (1.5) will also be discussed. In Section 3, we shall establish a sufficient condition assuring that (1.1) has a solution asymptotic to a given proper solution of (1.5) and show later (in Theorem 2) that this condition is also necessary under certain circumstances. Two corollaries involving simpler functionals $G$ will be given as examples to illustrate our main results. 


\section{DIFFERENCE EQUATIONS}

Consider the difference equation

$$
x(t)=G\left(t, x_{t}\right)+h(t),
$$

where $h \in C[0, \infty)$ and $G$ is a continuous functional defined as in Section 1 . The following result asserts that every solution of an initial value problem for (2.1) is proper.

LEMMA 1. If there exists a $K \in[0,1)$ such that for every $t \geqslant 0$ and $u, v \in C_{t}$

$$
|G(t, u)-G(t, v)| \leqslant K|u-v|,
$$

then for any $t_{0} \geqslant 0$ and any $u \in C\left[R\left(t_{0}\right), t_{0}\right]$ with $u\left(t_{0}\right)=G\left(t_{0}, u_{t_{0}}\right)+h\left(t_{0}\right)$ there exists a unique proper solution $x(t)$ satisfying (2.1) for $t \geqslant t_{0}$ and $x(t)=u(t)$ for $R\left(t_{0}\right) \leqslant t \leqslant t_{0}$.

Proof: It obviously suffices to show that for any $A>t_{0}$ there exists a unique solution $x(t)$ of (2.1) for $t_{0} \leqslant t \leqslant A$ with the given initial values.

Let $\rho>(1-K)^{-1}\left[\|u\|+\max _{t_{0} \leqslant t \leqslant A}\{|h(t)|+|G(t, 0)|\}\right]$ be a constant and let

$$
B_{\rho}=\left\{x \in C\left[R\left(t_{0}\right), A\right]: x(t)=u(t) \text { for } R\left(t_{0}\right) \leqslant t \leqslant t_{0}, \quad\|x\| \leqslant \rho\right\} .
$$

Evidently, $B_{\rho}$ is a bounded, closed, convex subset of the Banach space $C\left[R\left(t_{0}\right), A\right]$. Define an operator $T: B_{\rho} \rightarrow C\left[R\left(t_{0}\right), A\right]$ by

$$
(T x)(t)= \begin{cases}G\left(t, x_{t}\right)+h(t) & \text { for } t_{0} \leqslant t \leqslant A, \\ u(t) & \text { for } R\left(t_{0}\right) \leqslant t \leqslant t_{0} .\end{cases}
$$

Since for any $x \in B_{\rho}$,

$$
\begin{aligned}
\|T x\| & \leqslant\|u\|+\max _{t \leqslant t \leqslant A}\left\{|h(t)|+K\left\|x_{i}\right\|+|G(t, 0)|\right\} \\
& \leqslant(1-K) \rho+K \rho=\rho
\end{aligned}
$$

$T$ maps $B_{\rho}$ into itself. Also, it is easy to see from (2.2) that $T$ is a contraction on $B_{\rho}$. Hence, $T$ has a unique fixed point $x$ in $B_{\rho}$ which is the desired solution of (2.1) on $\left[R\left(t_{0}\right), A\right]$. This completes the proof of Lemma 1 .

We remark that in some specific cases Lemma 1 remains true without the assumption (2.2). For example, if $G\left(t, x_{t}\right)=g(t, x(t-r(t)))$ with $g$ continuous and $r(t)>0$, then the solution of any initial value problem of (2.1) can be obtained step by step and hence it is unique and proper. The following result gives some asymptotic properties of solutions to the difference equation

$$
x(t)=g(t, x(t-r(t)))+b t+q(t),
$$

where $r(t)>0, q \in C[0, \infty)$ and $b$ is a constant. We call the function $g(t, x)$ uniformly bounded in $t$ if $g(t, B)$ is uniformly bounded for $t \geqslant 0$ whenever $B$ is a bounded subset of $\boldsymbol{R}$. 
LEMMA 2. Suppose that $q(t)=o(t)$ as $t \rightarrow \infty, g(t, x)$ is uniformly bounded in $t$ and there exist constants $T>0, N>0$ and $K \in[0,1)$ such that

$$
|g(t, x)| \leqslant K|x| \text { for } t \geqslant T, \quad|x| \geqslant N .
$$

Then every solution $x(t)$ of $(2.3)$ satisfies

$$
\limsup _{t \rightarrow \infty} \frac{|x(t)|}{t} \leqslant|b| /(1-K) .
$$

Moreover, if $b \neq 0$, then the solution $x(t)$ also satisfies

$$
\liminf _{t \rightarrow \infty} \frac{x(t)}{b t} \geqslant 1 /(1+K) \text {. }
$$

If $b=0$ and $q(t)$ is bounded for $t \geqslant 0$, then $x(t)$ is bounded.

Proof: Let $x(t)$ be any solution of (2.3). As we mentioned before, $x(t)$ is a proper solution. Because of the uniform boundedness of $g(t, x)$ in $t$, there exists $M>0$ such that

$$
|g(t, x)| \leqslant M \text { for }|x| \leqslant N, \quad t \geqslant 0 .
$$

Then, from (2.3), (2.4) and (2.7) we have, for sufficiently large $t$,

$$
\begin{aligned}
|x(t)| / t & \leqslant|g(t, x(t-r(t)))| / t+|b|+o(1) \\
& \leqslant K|x(t-r(t))| / t+M / t+|b|+o(1) \leqslant K|x(t-r(t))| /(t-r(t))+|b|+o(1) .
\end{aligned}
$$

Letting $t \rightarrow \infty$ and noting that $t-r(t) \rightarrow \infty$, we obtain

$$
\limsup _{t \rightarrow \infty}|x(t)| / t \leqslant K \limsup _{t \rightarrow \infty}|x(t)| / t+|b| .
$$

Thus (2.5) follows immediately.

Similarly, we have, for all large $t$

$$
|x(t)| / t \geqslant|b|-K|x(t-r(t))| /(t-r(t))+o(1) .
$$

Again letting $t \rightarrow \infty$ we get

$$
\liminf _{t \rightarrow \infty}|x(t)| / t \geqslant|b| /(1+K) .
$$

If $b \neq 0$, then (2.8) implies that $|x(t)| \rightarrow \infty$, that is, either $x(t) \rightarrow+\infty$ or $x(t) \rightarrow-\infty$ as $t \rightarrow \infty$. Then there exists a sequence $t_{n}, t_{n} \rightarrow \infty$ as $n \rightarrow \infty$, such that $|x(t)| \leqslant$ $\left|x\left(t_{n}\right)\right|$ for $0 \leqslant t \leqslant t_{n}$, with $n=1,2, \ldots$. Since

$$
\left|g\left(t_{n}, x\left(t_{n}-r\left(t_{n}\right)\right)\right)\right| \leqslant K\left|x\left(t_{n}-r\left(t_{n}\right)\right)\right|+M \leqslant K\left|x\left(t_{n}\right)\right|+M,
$$


we see that $x\left(t_{n}\right)$ and $x\left(t_{n}\right)-g\left(t_{n}, x\left(t_{n}-r\left(t_{n}\right)\right)\right)$ have the same sign for all large $n$. But, in view of (2.3), the latter reaches the sign of $b$ eventually. Therefore $b x(t) \rightarrow+\infty$ and (2.6) follows from (2.8).

If $b=0$ and $|q(t)| \leqslant M_{1}$ for $t \geqslant 0$, then it follows from (2.3), (2.4) and (2.7) that

$$
|x(t)| \leqslant M+K|x(t-r(t))|+M_{1} .
$$

Assume, to the contrary, that $x(t)$ is unbounded, then there exists a sequence $t_{n} \rightarrow \infty$ such that $|x(t)| \leqslant\left|x\left(t_{n}\right)\right|, 0 \leqslant t \leqslant t_{n}, n=1,2, \ldots$, and $\left|x\left(t_{n}\right)\right| \rightarrow \infty$ as $n \rightarrow \infty$. But (2.9) leads us to

$$
\left|x\left(t_{n}\right)\right| \leqslant M+K\left|x\left(t_{n}-r\left(t_{n}\right)\right)\right|+M_{1} \leqslant M+M_{1}+K\left|x\left(t_{n}\right)\right|
$$

and hence

$$
\left|x\left(t_{n}\right)\right| \leqslant\left(M+M_{1}\right) /(1-K), \quad n=1,2, \ldots,
$$

a contradiction. Thus $x(t)$ is bounded, and the proof of Lemma 2 is complete.

\section{MAIN RESULTS}

Let $\phi(t) \in C\left[R\left(t_{0}\right), \infty\right)$ and $\delta>0$ be given for some $t_{0} \geqslant 0$. For convenience in the following discussions, we introduce the set

$$
H_{\phi}^{\delta}\left(t_{0}\right)=\left\{x \in C\left[R\left(t_{0}\right), \infty\right):|x(t)-\phi(t)| \leqslant \delta, \quad t \geqslant R\left(t_{0}\right)\right\} .
$$

The following theorem is our first result.

Theorem 1. Suppose that $\phi(t)$ is a proper solution of (1.5) for $t \geqslant t_{0} \geqslant 0$ and for some given $a$ and $b$. Suppose that there exist $K \in[0,1)$ and $\delta>0$ such that for any $t \geqslant t_{0}, x, y \in H_{\phi}^{\delta}(t)$

$$
\left|G\left(t, x_{t}\right)-G\left(t, y_{t}\right)\right| \leqslant K\left|x_{t}-y_{t}\right| .
$$

Assume further that there exists a nonnegative function $h \in C\left[t_{0}, \infty\right)$ (h may depend on $\phi$ and $\delta$ ) such that

$$
\left|F\left(t, x_{t}\right)\right| \leqslant h(t), \quad t \geqslant t_{0},
$$

for every $x \in H_{\phi}^{\delta}\left(t_{0}\right)$ and

$$
\int_{t_{0}}^{\infty} t h(t) d t<\infty
$$


Then there exists a solution $x(t)$ of (1.1) satisfying

$$
x(t)=\phi(t)+o(1) \text { as } t \rightarrow \infty
$$

Proof: Let $t_{1} \geqslant t_{0}$ be so large that $\int_{t_{1}}^{\infty} t h(t) d t \leqslant(1-K) \delta$. For any $x \in H_{\phi}^{\delta}\left(t_{1}\right)$, set $y=x-\phi$; then $y \in H_{0}^{\delta}\left(t_{1}\right)$. Obviously, $H_{0}^{\delta}\left(t_{1}\right)$ is a bounded, closed, convex subset of the Banach space $C_{\infty}\left[R\left(t_{1}\right), \infty\right)$ of all bounded continuous functions on $\left[R\left(t_{1}\right), \infty\right)$ equipped with the sup-norm. Define an operator $T: H_{0}^{\delta}\left(t_{1}\right) \rightarrow C_{\infty}\left(R\left(t_{1}\right), \infty\right)$ by the following relations

$$
\begin{aligned}
T y & =T_{1} y+T_{2} y \text { and } \\
\left(T_{1} y\right)(t) & = \begin{cases}G\left(t, y_{t}+\phi_{t}\right)-G\left(t, \phi_{t}\right), & t \geqslant t_{1}, \\
\left(T_{1} y\right)(t), & R\left(t_{1}\right) \leqslant t \leqslant t_{1},\end{cases} \\
\left(T_{2} y\right)(t) & = \begin{cases}\int_{t}^{\infty}(t-s) F\left(s, y_{s}+\phi_{s}\right) d s, & t \geqslant t_{1}, \\
\left(T_{2} y\right)(t), & R\left(t_{1}\right) \leqslant t \leqslant t_{1} .\end{cases}
\end{aligned}
$$

With the aid of (3.1), one can easily show that $T_{1}: H_{0}^{\delta}\left(t_{1}\right) \rightarrow H_{0}^{K \delta}\left(t_{1}\right)$ is a contraction. From (3.2), (3.3) and the choice of $t_{1}$ we have

$$
\left|\left(T_{2} y\right)(t)\right| \leqslant \int_{t}^{\infty} s h(s) d s \leqslant(1-K) \delta
$$

for $t \geqslant t_{1}$ and hence $T_{2}: H_{0}^{\delta}\left(t_{1}\right) \rightarrow H_{0}^{(1-K) \delta}\left(t_{1}\right)$. In view of (3.2), (3.3) and the continuity of $F, T_{2}$ is a continuous operator. It follows that $T: H_{0}^{\delta}\left(t_{1}\right) \rightarrow H_{0}^{\delta}\left(t_{1}\right)$ is continuous.

On the other hand, the assumptions on $F$ assert that all functions in $T_{2} H_{0}^{\delta}\left(t_{1}\right)$ are uniformly bounded, equicontinuous and equiconvergent to zero as $t \rightarrow \infty$. For any $\epsilon>0$, let $A>t_{1}$ be so large that $|y(t)|<\varepsilon / 2$ for every $y \in T_{2} H_{0}^{\delta}\left(t_{1}\right)$ and $t \geqslant A$. Since the restriction $S$ of $T_{2} H_{0}^{\delta}\left(t_{1}\right)$ to $\left[R\left(t_{1}\right), A\right]$ is precompact, there is a finite $(\varepsilon / 2)$-net $\left\{u_{1}, \ldots, u_{m}\right\}$ of $S$. Let $v_{i}(t)=u_{i}(t)$ for $R\left(t_{1}\right) \leqslant t \leqslant A$ and $v_{i}(t)=u_{i}(A)$ for $t \geqslant A$, $i=1, \ldots, m$. Then it is easy to show that $\left\{v_{1}, \ldots, v_{m}\right\}$ is an $\varepsilon$-net of $T_{2} H_{0}^{\delta}\left(t_{1}\right)$ in $C_{\infty}\left[R\left(t_{1}\right), \infty\right)$. Therefore, $T_{2} H_{0}^{\delta}\left(t_{1}\right)$ is precompact and hence $T_{2}$ is compact.

Thus, $T$ has a fixed point $y$ in $H_{0}^{\delta}\left(t_{1}\right)$ by the Krasnoselskii thoerem [3, Theorem 4.4.1]. Letting $x(t)=y(t)+\phi(t)$ for $t \geqslant R\left(t_{1}\right)$ and noting that $\phi(t)$ satisfies (1.5), we then obtain

$$
x(t)= \begin{cases}G\left(t, x_{t}\right)+a+b t-\int_{t}^{\infty}(s-t) F\left(s, x_{s}\right) d s, & t \geqslant t_{1}, \\ x\left(t_{1}\right)-\phi\left(t_{1}\right)+\phi(t), & R\left(t_{1}\right) \leqslant t \leqslant t_{1},\end{cases}
$$

a solution of (1.1). 
Finally, since

$$
\begin{aligned}
|x(t)-\phi(t)| & =\left|G\left(t, x_{t}\right)-G\left(t, \phi_{t}\right)-\int_{t}^{\infty}(s-t) F\left(s, x_{s}\right) d s\right| \\
& \leqslant K\left\|x_{t}-\phi_{t}\right\|+\int_{t}^{\infty} s h(s) d s, \quad t \geqslant t_{1},
\end{aligned}
$$

we can show, as in the proof of Lemma 2 , that

$$
\underset{t \rightarrow \infty}{\limsup }|x(t)-\phi(t)|=0
$$

which implies (3.4). This completes the proof of Theorem 1.

Next, we shall apply Theorem 1 to investigate the asymptotic linearity of solutions for the following NFDE

$$
[x(t)-p(t) x(t-\alpha-\beta t)]^{\prime \prime}+F\left(t, x_{t}\right)=0,
$$

where $\alpha \geqslant 0,0 \leqslant \beta<1, p \in C[0, \infty)$ and there is a constant $P,|P|<1$, such that $p(t)=P+o(1 / t)$ as $t \rightarrow \infty$.

COROllary. Let $\phi(t)=a+b t, a, b \in \mathrm{A}$. If there exist a $\delta>0$ and a nonnegative function $h \in C\left[t_{0}, \infty\right)$ for some $t_{0} \geqslant 0$ such that (3.2) and (3.3) hold for all $x \in$ $H_{\phi}^{\delta}\left(t_{0}\right)$, then the equation has a solution $x(t)$ satisfying

$$
x(t)=a+b t+o(1) \text { as } t \rightarrow \infty .
$$

ProOF: First of all, $\phi(t)$ satisfies

$$
\phi(t)-P \phi(t-\alpha-\beta t)=A+B t,
$$

where $A=a-P a+P b \alpha$ and $B=b-P b+P b \beta$. Next, let $y(t)$ be a proper solution of the difference equation

$$
y(t)-p(t) y(t-\alpha-\beta t)=A+B t
$$

((3.8) has a proper solution by Lemma 1$)$. We claim

$$
y(t)=\phi(t)+o(t) \text { as } t \rightarrow \infty
$$

In fact, from (3.7), (3.8) and the assumption on $p(t)$ we have

$$
\begin{aligned}
y(t)-\phi(t) & =p(t)[y(t-\alpha-\beta t)-\phi(t-\alpha-\beta t)]+(p(t)-P) \phi(t-\alpha-\beta t) \\
& =p(t)[y(t-\alpha-\beta t)-\phi(t-\alpha-\beta t)]+o(1) .
\end{aligned}
$$


Then, for some $K \in[0,1)$, and all large $t$, we get

$$
|y(t)-\phi(t)| \leqslant K|y(t-\alpha-\beta t)-\phi(t-\alpha-\beta t)|+o(1) .
$$

The same argument used in the proof of Lemma 2 allows us to affirm

$$
\limsup _{t \rightarrow \infty}|y(t)-\phi(t)|=0
$$

which gives (3.9).

Finally, Theorem 1 provides a solution $x(t)=y(t)+o(1)$ of $(3.5)$, which satisfies (3.6) by (3.9). This proves the corollary.

Now we turn to the following NFDE

$$
[x(t)-g(t, x(t-\tau(t)))]^{\prime \prime}+F\left(t, x_{t}\right)=0,
$$

where $g$ is continuous, $F$ is defined as before and $\tau \in C[0, \infty)$ with $0<\tau(t) \leqslant r(t)$. The related difference equation is

$$
x(t)=g(t, x(t-\tau(t)))+a+b t .
$$

Theorem 2. Let $g(t, x)$ be uniformly bounded in $t$. Suppose that there exist constants $T \geqslant 0, N \geqslant 0$ and $\alpha \in[0,1)$ such that

$$
|g(t, x)-g(t, y)| \leqslant \alpha|x-y| \text { for } t \geqslant T, \quad|x|, \quad|y| \geqslant N,
$$

and

$$
\begin{aligned}
\pm F(t, \pm u) & \geqslant 0, \\
\pm[F(t, \pm u)-F(t, \pm v)] & \geqslant 0
\end{aligned}
$$

for $t \geqslant T$ and for any $u, v \in C_{t}$ with $u(s) \geqslant v(s) \geqslant N,-r(t) \leqslant s \leqslant 0$. Then the equation (3.10) has a solution $x(t)=\phi(t)+o(t)$ as $t \rightarrow \infty$ for some proper solution $\phi(t)$ of (3.11) with $b \neq 0$ if and only if there exists a $k \neq 0$ such that

$$
\left|\int_{0}^{\infty} t F\left(t, y_{t}\right) d t\right|<\infty, \quad y(t)=k t
$$

Proof: Necessity. Let $\phi(t)$ be a proper solution of (3.11) with $b \neq 0$ and $x(t)=$ $\phi(t)+o(1)$ a solution of (3.10). Let us assume $b>0$. The case of $b<0$ can be dealt with in a similar way. In view of (3.12) and the uniform boundedness of $g(t, x)$ in $t$, we have

$$
\begin{aligned}
|g(t, x)| & \leqslant|g(t, x)-g(t, N)|+|g(t, N)| \\
& \leqslant \alpha|x|+\alpha N+|g(t, N)| \text { for } t \geqslant T, \quad|x| \geqslant N,
\end{aligned}
$$


which yields (2.4) for some $K \in(\alpha, 1)$. It follows from (2.6) in Lemma 2 that $\phi(t)$ tends to $+\infty$ as $t \rightarrow \infty$; so does $x(t)$. Choose $t_{0}>0$ so large that

$$
x(t) \geqslant N \text { for } t \geqslant R\left(t_{0}\right)
$$

Let

$$
w(t)=x(t)-g(t, x(t-r(t)))-\phi(t)+g(t, \phi(t-r(t)))
$$

then $w(t) \rightarrow 0$ as $t \rightarrow \infty$. Since, by (3.13) and (3.16),

$$
w^{\prime \prime}(t)=-F\left(t, x_{t}\right) \leqslant 0
$$

for $t \geqslant t_{0}$, we have $w^{\prime}(t) \geqslant 0, w(t) \leqslant 0$ and $w^{\prime}(t) \rightarrow 0$. Integrating the equation in (3.17) twice yields

$$
w(t)=-\int_{t}^{\infty}(s-t) F\left(s, x_{s}\right) d s, \quad t \geqslant t_{0}
$$

which implies

$$
\int_{t_{0}}^{\infty} t F\left(t, x_{t}\right) d t<\infty
$$

If we rewrite (3.18) as

$$
x(t)=g(t, x(t-r(t)))+b t+a-\int_{t}^{\infty}(s-t) F\left(s, x_{s}\right) d s,
$$

then from (2.6) we know that there is a $k \in(0, b /(1+K))$ such that $x(t)>k t$ for all large $t$, say, for $t \geqslant t_{1} \geqslant t_{0}$. We then appeal to (3.14) to conclude that

$$
\int_{t_{1}}^{\infty} t F\left(t, y_{t}\right) d t \leqslant \int_{t_{1}}^{\infty} t F\left(t, x_{t}\right) d t<\infty, \quad y(t)=k t
$$

which completes the necessity part of the proof.

Sufficiency. Again, we assume $k>0$ and omit the similar proof for $k<0$. Let $\phi(t)$ be any proper solution of (3.11) with $b=k(1-K) / 2$. By Lemma 2 we see that $\phi(t) \rightarrow+\infty$. Then we can choose a constant $t_{0} \geqslant T$ so large that for any $x \in H_{\phi}^{1}\left(t_{0}\right)$ we have

$$
N \leqslant x(t) \leqslant k t, \quad t \geqslant R\left(t_{0}\right) .
$$

Set $h(t)=F\left(t,(k \cdot)_{t}\right)$. Then (3.2) holds because of (3.14). Also, (3.15) gives (3.3). Thus, Theorem 1 provides a solution $x(t)=\phi(t)+o(1)$ of (3.10). This completes the proof of Theorem 2 . 
As an example, consider the equation

$$
\left[x(t)-|x(t-r)|^{\sigma} \operatorname{sgn} x(t-r)\right]^{\prime \prime}+\sum_{i=1}^{n} q_{i}(t) x\left(t-r_{i}(t)\right)=0,
$$

where $r>0, \sigma \in(0,1 / 2]$ are constants, $q_{i}, r_{i} \in C[0, \infty)$ are nonnegative functions with $t-r_{i}(t) \rightarrow \infty$ as $t \rightarrow \infty, i=1, \ldots, n$. The difference equation associated with $(3.20)$ is

$$
x(t)=g(x(t-r))+A+B t
$$

where $g(x)=|x|^{\sigma} \operatorname{sgn} x$. Applying Theorems 1 and 2 to the equation (3.20) we can deduce a stronger result.

Corollary. For any function of the form

$$
\psi(t)=a+b t+|b|^{\sigma} t^{\sigma} \operatorname{sgn} b, \quad b \neq 0, \quad t>0
$$

the equation (3.20) has a solution $x(t)=\psi(t)+o(1)$ as $t \rightarrow \infty$ if and only if

$$
\int_{0}^{\infty} t \sum_{i=1}^{\infty}\left(t-r_{i}(t)\right) q_{i}(t) d t<\infty
$$

Proof: We first observe that the function $g(x)$ is independent of $t$ and the functional $F\left(t, x_{t}\right)=\sum q_{i}(t) x\left(t-r_{i}(t)\right)$ is linear and hence (3.12-14) hold for, say, $T=0$, $N=1, \alpha=1 / 2$. We also note that (3.23) holds if and only if (3.15) holds for some, and hence all, $k \neq 0$. Therefore, all that is needed is that for every function $\psi(t)$ of the form (3.22) there exists a solution $\phi(t)$ of (3.21) such that $\phi(t)=\psi(t)+o(1)$ as $t \rightarrow \infty$. For if (3.23) holds, hence (3.15) holds for all $k \neq 0$, then we can find a solution $x(t)=\phi(t)+o(1)=\psi(t)+o(1)$ of (3.20), using the argument in the sufficiency part of the proof of Theorem 2. Conversely, if (3.21) has a solution $x(t)=\psi(t)+o(1)=\phi(t)+o(1)$, then Theorem 2 assures us of (3.15) for some $k \neq 0$, hence (3.23) holds.

To be definite, we assume that $b>0$. The discussion for $b<0$ proceeds similarly and is therefore omitted. Let $y(t)$ be a proper solution of (3.21) with $B=b$ and $A$ chosen properly. Lemma 2 implies that $y(t) \rightarrow+\infty$ as $t \rightarrow \infty$ and is bounded above by a linear function. It follows from (3.21) that

$$
y(t)=A+B t+o(t) \text { as } t \rightarrow \infty
$$

Substituting (3.24) into (3.21) we have

$$
y(t)=A+B t+(B t+o(t))^{\sigma}=A+B t+B^{\sigma} t^{\sigma}+o\left(t^{\sigma}\right) .
$$


Iterating (3.21) once more, we arrive at

$$
\begin{aligned}
y(t) & =A+B t+\left[A+B(t-r)+B^{\sigma}(t-r)^{\sigma}+o\left(t^{\sigma}\right)\right]^{\sigma} \\
& =A+B t+(B t)^{\sigma}\left[1+(B t)^{\sigma-1}+o\left(t^{\sigma-1}\right)\right]^{\sigma} \\
& =A+B t+(B t)^{\sigma}+\sigma(B t)^{2 \sigma-1}+o\left(t^{2 \sigma-1}\right)
\end{aligned}
$$

We now take $A=a$ if $\sigma<1 / 2$ and $A=a-\sigma$ if $\sigma=1 / 2$ so that $y(t)=a+b t+$ $(b t)^{\sigma}+o(1)=\phi(t)+o(1)$. This completes the proof of the corollary.

Remark. Apart from some manipulations, one can prove the corollary for $1 / 2<\sigma<1$ with a certain type of function $\psi(t)$ in place of (3.22); for example

$$
\psi(t)=a+b t+\operatorname{sgn} b \sum_{i=1}^{m} \sigma^{i-1}|b t|^{\sigma i-(i-1)}, \quad b \neq 0 \quad m \geqslant 1 \text { an integer }
$$

for $0<\sigma \leqslant m /(m+1)$. We omit the details.

\section{REFERENCES}

[1] S. Chen, 'Asymptotic linearity of solutions of nonlinear differential equations', Bull. Austral. Math. Soc. 35 (1987), 257-265.

[2] J. Hale, Theory of functional differential equations (Springer-Verlag, Berlin, Heidelberg, New York, 1977).

[3] D.R. Smart, Fixed point theorems (Cambridge Univ. Press, 1980).

Department of Mathematics,

Shandong University,

Jinan, Shandong, 250100

Peoples Republic of China 